\title{
Throughput Analysis of Receiver Initiated Collision Avoidance in Multi hop Wireless Networks
}

\author{
Rishi Pal Singh \\ School of Computer and \\ Systems Sciences \\ Jawaharlal Nehru University \\ New Delhi-110067
}

\author{
D.K.Lobiyal \\ School of Computer and \\ Systems Sciences \\ Jawaharlal Nehru University \\ New Delhi-110067
}

\begin{abstract}
This paper presents an analytical model based upon discrete time Markov chain analysis of receiver-initiated protocols for multi hop Ad hoc networks. Three-way receiver initiated (RTR-DATA-ACK) scheme for collision avoidance in Ad hoc networks has many protocols with it. In the proposed model, the nodes are randomly distributed according to a twodimension Poisson distribution with density $\lambda$. For the modeling, the effect of hidden terminals has been considered. As per the condition of saturation every node always has packet to transmit. The results show that the receiver-initiated collision avoidance scheme (proposed work) achieves higher throughput than the sender- initiated collision avoidance scheme for both the cases including short data packet as well as long data packet.
\end{abstract}

\section{General Terms}

Multi-hop Ad hoc Network, Hidden Terminal

\section{Keywords}

Adhoc netwoks, Markov Chain, Receiver Initiated Protocols

\section{INTRODUCTION}

Among many proposed scheme of collision avoidance many protocols of receiver initiated handshaking have been proposed in the past. The receiver initiated protocols are based upon three-way handshaking, which is RTR-DATA-ACK scheme between sender and receiver. Among all these protocols MACA-BI, RIMA-SP etc are some of the popular protocols $[11,12,16]$. Earlier the analytical models have been proposed for sender initiated protocols including IEEE 802.11 for WLAN $[1,2,6]$, but most of the analysis is for single hop networks. An analysis for multi-hop network has been done by $\mathrm{Yu}$ Wang el, who has proposed a simple multi-hop network model to derive the saturation throughput of a sender initiated collision avoidance scheme $[3,4,5,17]$. The receiver initiated protocols have been modeled in reference [18] but channel has not been modeled as per receiver initiated protocols. Moreover, the protocols used for the modeling are specific (RIMA-SP and RIMA-DP).

In the proposed work nodes are randomly distributed based upon the concept of Poisson distribution with density $\lambda$ [4]. The density of $\lambda$ will affect the level of congestion as well as the volume of hidden terminals. With the proposed model the saturation throughput analysis have been done of three way handshake general receiver initiated collision avoidance protocol. Therefore, the work of $\mathrm{Yu}$ el [17] Wang has is being extended for receiver initiated protocols.
In section 2 notations and assumptions for the modeling are discussed and section 3 to best of our knowledge, it is the first analytical model of receiver initiated collision avoidance in multi hop networks. In section 4 numerical results are discussed for the proposed model and compared with sender initiated analysis done by $\mathrm{Yu}$ Wang el [17]. It is also shown that the results are better in every case for our proposed model.

\section{PROPOPSED WORK}

\subsection{Notations and Assumptions}

1. In the proposed model, nodes are two dimensionally distributed with Poisson distribution of density $\square$, i.e., Prob (finding i terminals in an area of size $\mathrm{A})=\frac{(\lambda A)^{i}}{i !} e^{-\lambda A}$.

2. The packets with all the terminals always remain in a waiting state for their turn to be transmitted. It is only at the beginning of the slot, as in the case of slotted protocols, an effort is made to transmit a packet according to a Bernoulli process on the parameter $p$ where $0<p<1$.

\section{The traffic is assumed to be uniform.}

4. A fixed transmission power radius $R$ has been assumed for all the transmitters and receivers. Therefore, for any arbitrary terminal, the area of coverage is equal to $\pi R^{2}$. A terminal is defined to be a neighbor of another terminal, if they are within a distance $\mathrm{R}$ from each other.

5. A terminal while transmitting, can not receive simultaneously.

6. The probability of successful transmission is $p$ and not ready to transmit is $1-p$. The probability varies from one slot to another slot, subject to the current states of both the channel and the node.

These assumptions are there to make theoretical modeling good. For the simplification of analysis the channel is operated in time slotted manner because the maximum propagation delay is considerably lower than packet transmission time. In this case the performance of the slotted system will be same as of the un-slotted system. The length of each slot is denoted by $\tau$; this slot includes propagation delay plus other overheads. The transmission times of RTR and ACK packets are normalized w.r.t. $\tau$ and are denoted by $l_{r t r}$ and $l_{a c k}$. To simplify, we also assume that all packet 
transmission times are multiples of the length of a time slot [3, $5,13]$.

We also assume that in each slot, a silent terminal transmits a packet with probability $p \square$ and does not transmit with 1- $p \square$, where $p \square$ is defined to be

$\mathrm{p} \square=\mathrm{p}$ Prob. (the channel is sensed to be idle in a slot)

Here $\mathrm{p}^{\prime}$ is the transmission rate per slot for a terminal. Let $x$ be the terminal under consideration and channel around $x$ (or the channel that is to be used by terminal $\mathrm{x}$ be $\mathrm{CH}(\mathrm{x})$. Denote the limiting probability that $\mathrm{CH}(\mathrm{x})$ is sensed to be idle as $P c I$, then $p \square$ can be expressed as

$P^{\prime}=p \cdot P_{c I}$

For our analysis exact relation between $\mathrm{p}^{\prime}$ and $\mathrm{p}$ but a range of values of $\mathrm{p}^{\prime}$ is sufficient, because the throughput of protocols is influenced by the value of $p^{\prime}$. To find out the nearby relationship between $p$ and $\mathrm{p}^{\prime}$, we are modeling the channel with the help of Markov chain.

In the proposed model the channel is modeled as a circular region. The nodes within region have strong interaction and can communicate directly. These nodes have weak interaction with nodes of outside region. Because of weak interaction the transmitting node is almost unaffected by the activities of outer nodes and vice versa. $[3,5,17]$

\subsection{Approximate Performance Analysis}

To compute the transition probability, the method proposed by $[3,4,17]$ has been used. With the above assumptions the channel can be modeled with three state Markov chain as shown in figure1. The three states are idle, short and long and the significance of the states is as follow:

- Idle: The channel is in idle state when the channel around node $\mathrm{x}$ is sensed idle and the duration of this idle period is $\tau$. Thus

$$
T_{\text {idle }}=\tau
$$

- Long: The channel is in long state when a three way handshaking is done. The busy time for the channel in this state is

$$
\begin{aligned}
T_{\text {long }} & =l_{r t r}+\tau+l_{\text {data }}+\tau+l_{\text {ack }}+\tau \\
& =l_{r t r}+l_{\text {data }}+l_{\text {ack }}+3 \tau
\end{aligned}
$$

- Short: Short is the state for the collision of handshaking packet RTR. This collision of RTR takes place when multiple nodes around channel transmit simultaneously. The busy time for the channel in this state is

$$
\mathbf{T}_{\text {shotr }}=l_{r t r}+\tau
$$

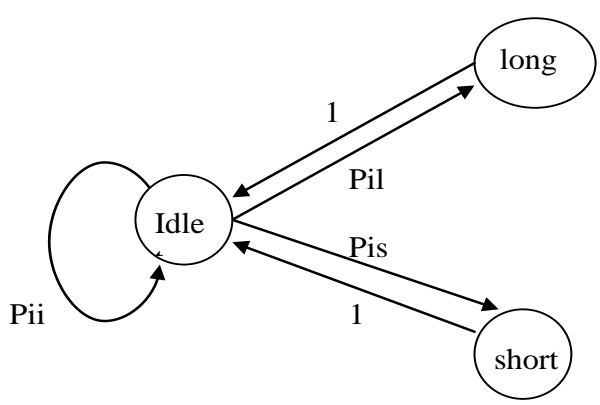

Figure 1: Markov chain model for the channel around a node.

As per the Poisson distribution of the nodes, the receiving range $\mathrm{R}$ of node $\mathrm{x}$ is having $\mathrm{i}$ nodes with probability $\frac{N^{i}}{i !} e^{-N}$ where $\mathrm{N}=\lambda \pi R^{2}$. Hence the mean number of nodes that belong to the shared channel is $M=\lambda \pi R^{\prime 2}=\alpha^{2} N$. We further assume that each node transmits independently and the probability of no transmission by any node is $\left(1-p^{\prime}\right)^{i}$. Here $\left(1-p^{\prime}\right)$ is the probability that a node does not transmit in a time slot.

The transition probability that channel transits from Idle to Idle state, denoted as $\mathrm{P}_{\mathrm{ii}}$ can be expressed as

$$
\begin{aligned}
\mathrm{P}_{\mathrm{ii}} & =\sum_{i=0}^{\infty}\left(1-p^{\prime}\right)^{i} \frac{M^{i}}{i !} e^{-M} \\
& =e^{-p^{\prime} M}
\end{aligned}
$$

Next is the calculation of transition probability from Idle state to Long state, denoted as $\mathrm{P}_{\mathrm{il}}$. If there are $i$ nodes around channel $\mathrm{x}$, for a successful transmission one successful three way handshake has to take place for single node while other nodes do not transmit. Let $\mathrm{p}_{\mathrm{s}}$ denote the probability that a node begins a successful three-way handshake at each slot $[3,17]$.

$\mathrm{P}_{\mathrm{il}}$ can be calculated as follows

$$
\begin{aligned}
& =\sum_{i=1}^{\infty} i p_{s}\left(1-p^{\prime}\right)^{i-1} \frac{M^{i}}{i !} e^{-p^{\prime} M} \\
& =p_{s} M e^{-p^{\prime} M}
\end{aligned}
$$

We have calculated $\mathrm{P}_{\mathrm{ii}}, \mathrm{P}_{\mathrm{il}}$. We can calculate $\mathrm{P}_{\text {is }}$, the transition probability from idle to short, which is equal to $1-\mathrm{P}_{\mathrm{ii}}-\mathrm{P}_{\mathrm{il}}$. Let $\pi_{i}, \pi_{l}$ and $\pi_{s}$ denote the steady state probabilities of states idle, long and short respectively.

$$
\begin{aligned}
& \pi_{i} p_{i i}+\pi_{l}+\pi_{s}=\pi_{i} \\
& \pi_{i} p_{i i}+1-\pi_{i}=\pi_{i} \\
& \pi_{i}=\frac{1}{2-p_{i i}}=\frac{1}{2-e^{-p^{\prime} M}}
\end{aligned}
$$

Now we calculate the limiting probability for the channel found idle: 


$$
\begin{aligned}
P_{C I} & =\frac{\pi_{i} T_{i d l e}}{\pi_{i} T_{\text {idle }}+\pi_{l} T_{\text {long }}+\pi_{\text {short }} T_{\text {short }}} \\
& =\frac{T_{\text {idle }}}{T_{\text {idle }}+P_{i l} T_{\text {long }}+P_{i s} T_{\text {short }}}
\end{aligned}
$$

The relationship between $p^{\prime}$ and $p$ is then

$$
p^{\prime}=\frac{p T_{\text {idle }}}{T_{\text {idle }}+P_{i l} T_{\text {long }}+P_{i s} T_{\text {short }}}
$$

In the above equation probability of success to transmit three way handshake in a time slot, $p_{s}$, is yet to be determined. Now we determine the probability of successful transmission from node $x$ in a slot. This is equal to throughput by definition. We model the states of node $x$ by a three state Markov chain as shown in figure 2 . The three states of the chain are

- Wait: When the node defers for other nodes or backs off.

- Successful: This is the state of the node when it successfully completes three-way handshake.

- Collision: This is the state of the node when there is collision during handshake.

The length of the time the node $x$ spends at any state will be as follow:

$T_{\text {succ }}=T_{\text {long }}$

$T_{\text {coll }}=T_{\text {short }}$

$T_{\text {wait }}=\tau$

For the collision avoidance we also assume that when node $x$ finishes transmission, it will not transmit immediately following the previous transmission; therefore, the transition probabilities from success to wait and from collision to wait are 1 .

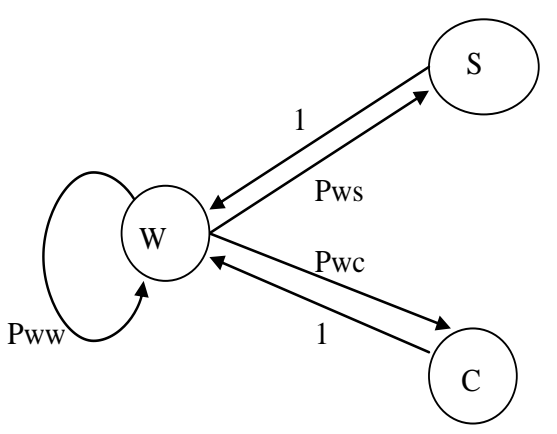

Figure 2: Markov chain model for a node

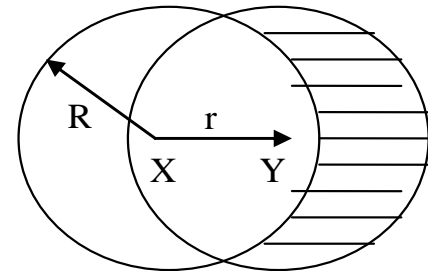

Figure 3. Illustration of "hidden" nodes and communication in multi-hop

For derivation of the transition probability $p_{w s}$,we need to derive the probability $p_{w s}(r)$ that node $\mathrm{x}$ successfully initiates a three-way handshake with node $\mathrm{y}$, where $\mathrm{r}$ is the distance between $\mathrm{x}$ and $\mathrm{y}$. Before calculating $p_{w s}(r)$, we define $\mathrm{B}(r)$ to be the area that is in the range of node $y$ but outside the range of node $x$ as the shaded area of the figure.

$$
B(r)=\pi R^{2}-2 R^{2} q\left(\frac{r}{2 R}\right)
$$

where

$$
q(t)=\arccos (t)-t \sqrt{1-t^{2}}
$$

Then $P_{w s}$ can be calculated as:

$$
p_{w s}(r)=p_{1} \cdot p_{2} \cdot p_{3} \cdot p_{4}(r)
$$

$p_{w s}(r)=\operatorname{Prob}(x$ transmits in a slot $)$. Prob $(y$ does not transmit in the same slot). Prob (none of the terminals within $R$ of $x$ transmits in the slot). Prob (none of the terminals in $\mathrm{B}(r)$ transmits for $\left(2 l_{r t r}+1\right)$ slots $\left.\mid \mathrm{r}\right)$

$$
\begin{aligned}
& p_{1}=\operatorname{Prob}(\mathrm{x} \text { transmits in a slot) } \\
& p_{2}=\operatorname{Prob}(\mathrm{y} \text { does not transmit in the same slot) } \\
& p_{3}=\operatorname{Prob} \text { (none of the terminals within } R \text { of } x \text { transmits in } \\
& \text { the slot) }
\end{aligned}
$$

$p_{4}(r)=$ Prob (none of the terminals in $\mathrm{B}(r)$ transmits for $\left(2 l_{r t r}+1\right)$ slots $\left.\mid r\right)$

Obviously, $p_{1}=p^{\prime}$ and $p_{2}=\left(1-p^{\prime}\right) \cdot p_{3}$ can be obtained by

$$
\begin{aligned}
p_{3} & =\sum_{i=0}^{\infty}\left(1-p^{\prime}\right)^{i} \frac{\left(\lambda \pi R^{2}\right)^{i}}{i !} e^{-\lambda \pi R^{2}} \\
& =e^{-p^{\prime} N}
\end{aligned}
$$


Similarly, the probability that none of the terminals in $B(r)$ transmits in a time slot is given by

$$
\begin{aligned}
p_{4}(r) & =\sum_{i=0}^{\infty}\left(1-p^{\prime}\right) \frac{(\lambda B(r))^{i}}{i !} e^{-\lambda B(r)} \\
& =e^{-p^{\prime} \lambda B(r)}
\end{aligned}
$$

Hence $p_{4}(r)$ can be expressed as

$$
\begin{aligned}
p_{4}(r) & =\left(p_{4}(r)\right)^{2 l_{r t r}+1} \\
& =e^{-p^{\prime} \lambda B(r)\left(2 l_{r t r}+1\right)}
\end{aligned}
$$

The probability density function of the distance $r$ between node $\mathrm{x}$ and $\mathrm{y}$ is

$$
f(r)=2 r, \quad 0<r<1
$$

where we have normalized $\mathrm{r}$ with regard to $\mathrm{R}$ by setting $R=1$. Now we can calculate $p_{w s}$ as follows:

$$
\begin{aligned}
p_{w s} & =\int 2 r p_{w s}(r) d r \\
& =2 p^{\prime}\left(1-p^{\prime}\right) e^{-p^{\prime} N} \int_{0}^{1} r e^{-p^{\prime} \lambda B(r)\left(2 l_{r r}+1\right)} d r \\
& =2 p^{\prime}\left(1-p^{\prime}\right) e^{-p^{\prime} N} \int_{0}^{1} r e^{-p^{\prime} N[1-2 q(r / 2) / \pi]\left(2 l_{r t r}+1\right)} d r
\end{aligned}
$$

From figure, the transition probability $p_{w w}$ that the node stays in wait state in a slot is $\left(1-p^{\prime}\right) e^{-p^{\prime} N}$ means node $\mathrm{x}$ does not initiate any transmission and there is no node around it initiating a transmission.

Let $\pi_{w}, \pi_{s}$ and $\pi_{c}$ denote the steady-state probability of state success, wait and collision respectively. From figure, we have

$$
\begin{gathered}
\pi_{w} p_{w w}+\pi_{s}+\pi_{c}=\pi_{w} \\
\pi_{w} p_{w w}+1-\pi_{w}=\pi_{w} \\
\pi_{w}=\frac{1}{2-p_{w w}} \\
=\frac{1}{2-\left(1-p^{\prime}\right) e^{-p^{\prime} N}}
\end{gathered}
$$

Therefore, the steady-state probability of state success $\pi_{s}$ can be calculated as:

$$
\pi_{s}=\pi_{w} p_{w s}=\frac{p_{w s}}{2-\left(1-p^{\prime}\right) e^{-p^{\prime} N}}
$$

Thus the throughput $T h$ is:

$$
T h=\frac{\pi_{s}}{\pi_{w} T_{w}+\pi_{s} T_{s}+\pi_{c} T_{c}}
$$

\section{NUMERICAL RESULTS}

In this section, we compare the throughput of receiver initiated scheme with sender initiated scheme. Here $\tau$ represents the duration of one slot time. The length of RTR, RTS, CTS and ACK packets is $5 \tau$. Based upon the number of nodes in the region $(\mathrm{N}=3, \mathrm{~N}=6$ and $\mathrm{N}=10)$ the performance of receiver initiated and sender initiated scheme is compared. The figure plots throughputs versus attempt probability $p$ '. The throughput decreases as the number of nodes in the region increases. The performance of receiver initiated protocols is better than sender initiated protocols. The performance of the protocols is compared with two cases of data size. The size of data taken for short data is $20 \tau$ and the size of the long data size is $100 \tau$. The throughput is better for long data size as compared to the short data size.

Initially the throughput increases with the attempt probability $p^{\prime}$ than degrades rapidly. A close look at figure reveals that the value of attempt probability $p$ that achieves maximum throughput is very low.

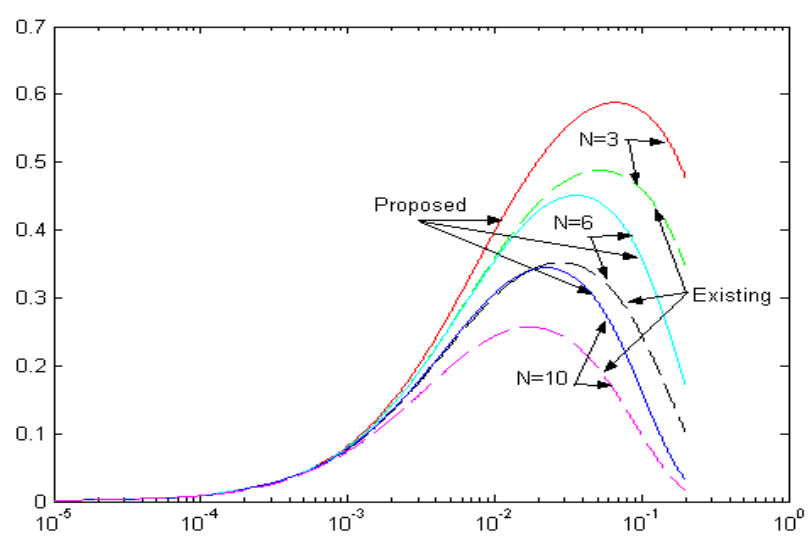

(a) Long Data Packet $l_{\text {data }}=100 \tau$

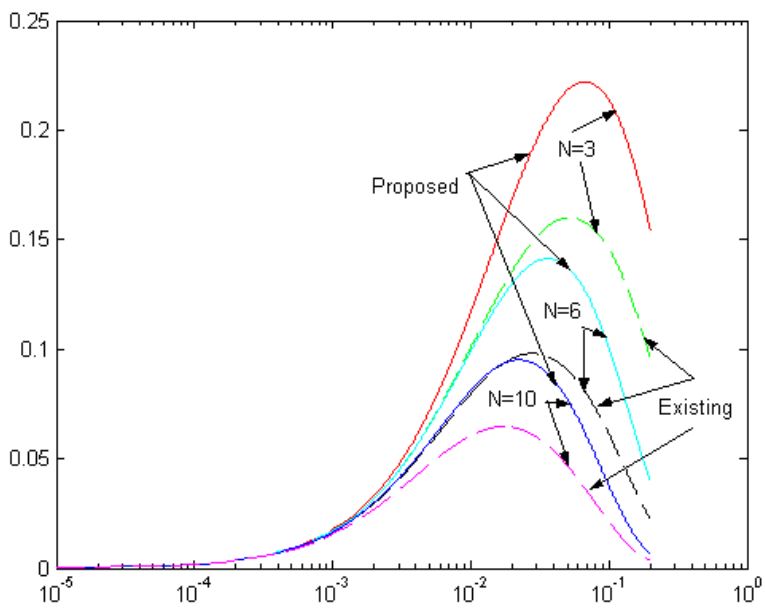

(b) Short Data Packet $l_{\text {data }}=20 \tau$

Figure 4. Throughput Comparison of Receiver initiated v/s Sender initiated Schemes 


\section{CONCLUSIONS}

In this paper, a model has been proposed to compute the saturation throughput of receiver initiated protocols based upon RTR-DATA-ACK handshake in multi hop Ad hoc networks. The results indicate that the performance of receiver-initiated collision avoidance is much better than the sender-initiated collision avoidance. The saturated throughput is being computed by means of Markov chain model. The results also indicate that the performance of the protocol is better for long packet size data than the short packet size data. The throughput decreases as the number of nodes in the region increases.

\section{REFERENCES}

[1] Bianchi, G., 2000 Performance analysis of the IEEE 802.11 Distributed Coordination Function IEEE journal on selected Area in Communication, 18(3):535-547.

[2] Bianchi, G., Fratta, L., and Oliveri M., 1996. Performance Evaluation and Enhancemaent of the CSMA/CA MAC Protocol for IEEE 802.11 WLAN. In Proceeding of PIMRC '96, pages 392-396.

[3] Wang, Yu., Garcia-Luna-Aceves, J.J., 2004. Modeling of Collision Avoidance Protocols in Single-Channel Multihop Wireless Networks. Wireless Networks, Vol. 10 No. 5, 495-506.

[4] Takagi, H., and Kleinrock, L., 1984 Optimal Transmission Range for Randomly Distributed Packet Radio Terminals. IEEE Transactions on Communication. 32(3):246-257.

[5] Wu, L., and Varshney P., 1999. Performance Analysis of CSMA and BTMA Protocols in Multihop Networks(I) Single Channel Case. Information Sciences, Elsevier Sciences Inc., 120:159-177.

[6] Chhaya, H.S. and Gupta S., 1996. Performance of Asynchronous Data Transfer Methods of IEEE 802.11 MAC Protocol", IEEE Pers. Comm., 3(5).

[7] Tobagi, F., 1987. Modeling and Performance Analysis of Multihop Packet Radio Networks. Proceeding of IEEE vol 75, No. 1, 135-155.

[8] Ozugur, T.,.Naghshineh, M., Kermani P., and.Copeland J.A 1999. Fair Media Access for Wireless LANs. In Proceeding of IEEE GLOBECOM'99.

[9] Tobagi, F.A. and Kleinrock, L. 1975. Packet switching in radio channels: Part II - the hidden terminal problem in carrier sense multiple-access modes and the busy-tone solution. IEEE Trans Comm vol. COM-23, no. 12, pp. $1417-1433$.

[10] Trivedi K.S. 2007. Probaaility and Statistics with Reliability,Queueing and Computer Science Applications. Prentice Hall of India.

[11] Karn, P. 1990. MACA- a new channel access method for packet radio. In ARRL/CRRL Amateur Radio $9^{\text {th }}$ Computer Networking Conference pp. 134-40.

[12] Tallucci, F. and.Gerla, M. 1997. MACA-BI (MACA by Invitation) : A wireless MAC protocol for high speed ad hoc Netwoking," ICUPC

[13] Fullmer, C.L. and Garcia-Luna-Aceves, J. 1995. Floor Acquisition multiple Access (FAMA) for packet-radio Networks. In SIGCOMM 1995 pages 262-273. ACM August 1995.Cambridge MA (USA).

[14] Tobagi, F.A. and Kleinrock, L. 1975. Packet switching in radio channels: Part I- carrier sense multiple-access modes and their throughput delay characteristics. IEEE Trans Comm vol. COM-23, no. 12, pp. 1400-1416.

[15] Rom, R. and Sidi, M. 1990. Multiple Access Protocols performance and analysis. Springer-verlog.

[16] Garcia-Luna-Aceves, J. and Tzamalous A. 2002. Receiver-Initiated Collision Avoidance in Wireless Networks. Wireless Networks, 8, 249-263.

[17] Wang, Yu., Garcia-Luna-Aceves, J.J. 2002. Collision Avoidance in Multi-Hop Ad Hoc Networks. In proc of IEEE MASCOTS Oct 2002 pp 145-154 Washington DC.

[18] Moraes, R. and García-Luna-Aceves, J.J. 2004 "Receiver-Initiated Collision Avoidance in Multi-hop Ad Hoc Networks. In Proceedings of International Conference on Communications in Computing (CIC'04), Monte Carlo Resort, Las Vegas, Nevada, USA. 